\title{
DECOMPOSITION KINETICS OF MATERIALS COMBINING BIOMASS AND ELECTRONIC WASTE
}

\author{
Juan A. Conesa*, Aurora Soler \\ Department of Chemical Engineering, University of Alicante, P.O. Box 99, E-03080 Alicante \\ *Email: ja.conesa@ua.es
}

\begin{abstract}
The disposal of waste from electrical and electronic equipment (WEEE) is an actual problem of industrialized countries. In the present work, thermal decomposition of different materials has been studied in a thermobalance at different heating rates. Kinetic models are proposed for the pyrolysis, gasification and combustion of crushed wood pellets (CWP), halogen-free electrical wires (EWs) and printed circuit boards (PCBs). Three different heating rates were used at each atmosphere condition. One set of parameters can explain all the experiments at the different atmospheres and at the three different heating rates used. In the case of CWP, a model considering three independent first-order reactions gave very good correlations all the heating rates tested both in inert and oxidant atmosphere. The decomposition of synthetic materials (EW and PCB) is modelled by using n-th order kinetics. On the other hand, mixtures of these three materials have been prepared and tested for decomposition behaviour. A weighted sum of the curves simulated using kinetics of the materials separately gives a good concordance with the experimental curve in the case of PCBs, indicating that there is not chemical interaction between CWP and PCBs when heated and decomposed. This would indicate that the production of pollutants in the decomposition will not be affected by the presence of the other material. Nevertheless, a strong interaction is found with the mixtures between biomass and EW.
\end{abstract}

Keywords: combustion, pyrolysis, printed circuit boards, electrical wire, thermogravimetry, kinetic parameters

\section{Introduction}

In the most recent years, the presence of electrical and electronic equipment has increased significantly in our daily lives because they simplify chores and are essential in the workplace. Practically these equipment appear in all areas of human activity in developed areas, and great technological advances cause that this type of equipment become obsolete in a short time and be quickly replaced by new equipment, thus becoming a growing source of waste.

According to the report of the United Nations University (UNU), 41.8 million tonnes of waste from electrical and electronic equipment (WEEE) were generated worldwide in 2014 [1]. This type of equipment is composed of many different products. From simple and easily recyclable raw materials such as plastics and base metals (copper, tin, steel), to rare earth or precious metals (gold, silver), and hazardous products such as cadmium, mercury and lead. Therefore, these wastes are considered a serious threat to the human health and the environment. 
The mixture of different materials in the waste makes difficult its management and becomes an environmental challenge of the highest order. In this sense, government directives pretend the reuse, recycling and prevention of the generation of WEEE, as it can be seen in the latest Electrical and Electronic Equipment Waste Directive in the European Union (Directive 2012/19/EU).

Among these wastes, electric wires and printed circuit boards are especially problematic to recycle because of great variety of organic materials and metals. The first stage of recycling is to separate metals and plastics by physical and magnetic techniques, given that metal is the most valuable fraction of these wastes. In electrical wires, approximately $50 \%$ by mass is copper []ㅡ, while metal fraction is $30 \%$ by mass in PCBs formed by copper, tin, lead, iron, nickel and noble metals []ㅡ. Once the metals are separated, the plastic fraction has been traditionally a product with low value, but nowadays is well considered it suitable for recycling, by material, chemical or energy recovery. Thermal disposal, especially incineration, is a desired and a viable alternative for plastic waste and is often used in industrialized countries, but is on the back foot of a more popular option, landfilling which is at present the most common disposal method, although contrary to the guidelines of the new rules [4]].

The most problematic component in the cable plastic waste is the PVC fraction. In a previous work [ $\underline{5}$ ], emission tests were performed on the thermal decomposition of PVC and halogen-free electrical wires, pointing out the important quantities of poly-chlorinated dioxins and furans emitted in uncontrolled processes. On the contrary, a reduced amount of pollutants was measured in the halogen-free electrical wires thermal decomposition.

On the other hand, PCB wastes are also growing as a consequence of increasing the use of mobile phones and computers, among others. In this sense, about 65-80 per cent of the material content of a mobile phone can be recycled and reused. The first stage of recovery is separation of the shredded metals into different fractions. Different metals are sold to metal refineries, which ends with a plastic residue with difficult treatment. Due to their high thermal value, the thermal treatment of plastics can be an attractive approach to recycle these wastes.

Thermogravimetric (TG) analysis is based on measuring the change in mass of a sample when this sample is subjected to a temperature program in a controlled atmosphere. This technique has been widely used to study the thermal decomposition of different waste electrical and electronic equipment, given that the TG curves provide information on the mechanisms of decomposition of the sample and allow for calculate the kinetic parameters of the reaction that has taken place []ㅡ.

There are interesting contributions in literature considering pyrolysis and/or combustion of WEEE and biomass wastes, proposing kinetic models that simulate the behavior of the raw materials studied in the present work. Gronli et al. [ 6 ] used TG analysis to study the pyrolysis of nine wood species and obtain the kinetic parameters of each of species. Ortuño et al. [7] studied the thermal decomposition of waste printed circuit board and proposed a kinetic model for combustion and pyrolysis of this material. Conesa et al. [ $\underline{5}$ obtained kinetic parameters for thermal decomposition of PVC and halogen-free electric wires in different atmospheres (pyrolysis, combustion 4:1, combustion 9:1). An interesting kinetic analysis of PVC (waste and virgin material) decomposition in a helium atmosphere was made [ $\underline{8}]$ using a model constituted by several competitive (parallel) and/or consecutive reactions. Benes et al. [9] determined the thermal behavior of PVC cable insulation in forms as supplied and as chemically treated with organic solvents to remove plasticizers.

On the other hand, Dong et al. [10] studied pyrolysis of different mixtures of poly-ethylene (PE) and wood and determined that the composition of the evolved light gas depended on the interaction between PE and wood, but there was not any effect on overall kinetics. Also, Kim et al. [11] studied the pyrolysis of different mixtures of PE and wood 
and did not find mixture effects on kinetics. In this way, the results reported in the literature confirm negligible effects of interactions on kinetics. In the present paper, a kinetic model for the thermal decomposition of CWP (biomass) and two different WEEE (halogen-free electrical wires and printed circuit boards) is proposed. Furthermore, mixtures of these three materials have been prepared and tested for decomposition behavior and their behavior is discussed.

2. Experimental Section

\subsection{Materials}

\subsubsection{Biomass (CWP)}

Wood is one of the main sources of heat and energy. It is used as alternative energy by thermal processing (pyrolysis, combustion and gasification), given that it has much more advantages that other biomass, for example, higher energy content per volume, lower amount of ash and a low amount of nitrogen and sulfur.

In the present study, CWP was obtained after grinding commercial pellets, which were manufactured and supplied by Ertasa in the form of cylinders of compacted biomass from forest residues and waste from plants.

All of the materials used in the present work were grinded in a vibratory disc mill RETSCH RS 200 and then were grinded in cryogenic ball mill (RETSCH CryoMill) to diminish the diameter of the particles until $<0.1 \mathrm{~mm}$.

Elemental analysis of the biomass feedstock was performed in Thermo Finnigan Flash 1112 Series elemental microanalyzer. Oxygen plus ash content is determined by difference. Furthermore, ash content was determined by mass loss at $550^{\circ} \mathrm{C}$ following norm UNE-EN-14775:2009. The concentration of other elements was measured using a model PW2400 automatic sequential spectrometer X-ray fluorescence. Table 1 shows the results of these analyses.

[Table 1]

\subsubsection{Electrical wire (EW)}

The halogen-free electrical wire employed in the present work was supplied by General Cable, Co. It is a special fireresistant and halogen-free wire. The material used in the cover (or jacket) is a mixture of polyethylene, a thermoplastic elastomer, and aluminum trihydroxide (PE-TPE-ATH) and for the insulation the material used is a silicone rubber.

Prior to the thermogravimetric analysis, the plastic fraction was separated of metallic fraction by a mechanic technique, given that in the present study only the plastic fraction was used. The cover and insulation were shredded to fine dust (fraction under $0.1 \mathrm{~mm}$ ). Composition and elemental analysis of the cover and the insulation were also determined and is shown in Table 1.

In the mathematical treatment of the decomposition data, EW has been treated as a mixture of two decomposing fractions, cover and isolation, that does not interact, as was already shown [ $\underline{5}]$.

\subsubsection{Printed circuit boards (PCBs)}

Actually, there are two main types of PCBs in WEEE: paper-based phenolic resin PCBs and fiberglass-based epoxy PCBs. In the present study, PCBs used were fiberglass-based epoxy resin before its use in the manufactures of circuit boards, in order to be sure of the absence of metals. This material was supplied by CISA, Circuitos Impresos S.A. In particular, FR-4 PCBs were used in the present work, which are created from the different materials (epoxy resin, woven glass fabric reinforcement, brominated flame retardant, etc.) and were crushed to $<0.1 \mathrm{~mm}$.

Table 1 also shows the results of the composition and elemental analyses of the PCB used in the present work. It is important to point out the high concentration of bromine in this material, from the flame-retardants used in its 
manufacturing and that has been proved to be responsible for the emission of brominated pollutants during its thermal decomposition [12].

\subsection{Sample preparation}

Besides of the materials separately, four mixtures were prepared mixing CWP with halogen-free electrical wires (EWs) and printed circuit boards (PCBs). The completed mixtures were 20 and $50 \%$ of EW in CWP and also 20 and $50 \%$ of PCBs in CWP. The samples were mixed in the convenient proportion and were homogenized by mixing mechanically during at least one hour.

\subsection{Thermo-gravimetric analysis (TGA)}

Runs for the TG analysis were carried out on a Mettler Toledo TGA/SDTA851e/SF/1100 Thermal Gravimetric Analyzer. The decomposition temperatures were measured under dynamic conditions in different atmospheres such as nitrogen and mixtures of nitrogen and oxygen $\left(4: 1\right.$ and 9:1) with a total flow rate of $100 \mathrm{~mL} \mathrm{~min}^{-1}$. Non isothermal dynamic experiments were carried out at heating rates of 5, 10 and $20 \mathrm{~K} \mathrm{~min}^{-1}$ for each atmosphere, from room temperature up to $1173 \mathrm{~K}$. For each experiment $8 \pm 0.3 \mathrm{mg}$ of sample were used. Duplicated runs in the TG were done in order to test for the reproducibility.

\section{Kinetic models and optimization method}

The kinetic model proposed for thermal decomposition in the three atmospheres of materials studied could be interpreted considering each material formed by two or three independent parts (depending on the material), each one following an independent reaction, as follows:

$$
c_{\text {Si0 }} \text { Solid }_{i} i-\text { th reaction }_{\rightarrow}\left(c_{S i 0}-v_{i \infty}\right) \text { Residue }_{i}+v_{i \infty} \text { Volatiles }_{i}
$$

With $\mathrm{i}=1-3$ for biomass CWP and $\mathrm{i}=1-2$ for PCBs and parts of the EWs (details will be discussed later). In the previous equation, $\mathrm{Solid}_{\mathrm{i}}$ refers to different fractions of the original material, Volatiles $\mathrm{i}_{\mathrm{i}}$ are the gases and condensable volatiles evolved in the corresponding reactions and Residue $\mathrm{i}_{\mathrm{i}}$ is the possible residue formed in the decomposition of each Solid . The small letters represent the yields coefficients representative of each reaction and, consequently, it is considered that they do not change with time or with the extension of reaction. Each fraction has a yield coefficient that represents the maximum mass fraction obtainable by each reaction. In this way, $v_{i \infty}$ is the yield coefficient for the Volatiles ${ }_{\mathrm{i}}$ and $\left(c_{S i 0^{-}}\right.$ $\left.v_{i \infty}\right)$ is the yield coefficient for the Residue $\mathrm{i}_{\mathrm{i}}$. On the other hand, the sum of initial mass fractions of the components $\left(c_{S i 0}\right)$ is exactly one minus average value of the final mass fractions of solid of all heating rates considered []].

The conversion degree for each reaction is defined as the ratio between the mass fractions of solid reacted at any time $\left(c_{S i 0}-w_{S i}\right)$ and the corresponding initial fraction of this component.

$$
\alpha_{i}=\frac{c_{S 0 i}-w_{S i}}{c_{S i 0}}, i=1 \dot{\iota} 3
$$

From the mass balance between products and reactants and the conversion degrees, the kinetic equations for pyrolysis and combustion runs can be defined as follows: 


$$
\frac{-d\left(\frac{w_{S i}}{c_{S i 0}}\right)}{d t}=k_{i}\left(\frac{w_{S i}}{c_{S i 0}}\right)^{n_{i}} \vee \frac{d \alpha_{i}}{d t}=k_{i}\left(1-\alpha_{i}\right)^{n_{i}}
$$

with $n_{i}$ being the reaction order and the kinetic constants following the Arrhenius equation:

$$
k_{i}=k_{i 0} \exp \left(\frac{-E_{i}}{R T}\right)
$$

In the case of biomass (CWP) decomposition, both in absence (pyrolysis) and in the presence of oxygen (gasification, combustion), the reaction orders obtained have been maintained as unity, for all the fractions considered. This has been pointed out previously in different studies $[\underline{6}, \underline{13}, \underline{14}]$ where it is clear that using this structural model based on the chemical components (cellulose, hemicellulose and lignin) it is valid a first order approximation.

For calculation of the total mass remaining a weighted sum is used:

$$
\frac{-d w_{s}^{c a l}}{d t}=\sum_{i}^{\square} c_{S i 0} \frac{d \alpha_{i}}{d t} \wedge w_{s}^{c a l}=1-\sum_{i}^{\square} c_{S i 0} \alpha_{i}
$$

For the optimization of 9 TG runs ( 3 heating rates $\times 3$ different atmospheres), 11 parameters were calculated considering three fractions $\left(3 \times E_{\mathrm{i}}, 3 \times k_{\mathrm{i}}, 3 \times n_{\mathrm{i}}\right.$ and $\left.2 \times c_{\mathrm{Si}}\right)$. In the case of materials where only two fractions were necessary, only 7 parameters were optimized; if there was considered one fraction the number of parameters was 3 . The optimization was done by integration of the differential equations presented in the kinetic model by the Euler method considering and testing that the intervals of time were small enough to make the integration errors negligible. The optimization method of the function Solver in a Microsoft Excel spreadsheet was used to minimize the differences between the experimental and calculated mass loss and their derivatives. The objective function (OF) to minimize was the sum of the square differences between the experimental and calculated mass loss and their derivatives multiplied by a factor ' $f$ ':

$$
\begin{gathered}
\sum_{p=1}^{c a l}\left(i w_{S m, p}^{\exp }\right)^{2}+f \cdot \sum_{m=1}^{M} \sum_{p=1}^{P}\left(\frac{d w_{S m, p}^{c a l}}{d t}-\frac{d w_{S}^{\exp }}{d t}\right)^{2} \\
\sum_{m=1}^{M} i \\
O F=i
\end{gathered}
$$

where where ' $p$ ' represents the experimental data at time ' $t$ ' in the experiment with a heating rate ' $m$ '. The value of $M$ is the number of runs and $P$ is the number of points in each run. The value of ' $f$ ' was arbitrarily chosen to be $10^{+3}$, in order to give similar contribution to the OF to the mass loss differences and those of the derivatives. Note that with this methodology, a unique set of kinetic constants is calculated from the experimental curves obtained at different heating rates, and it gives kinetic constants valid for the whole set of heating rates used.

The points represented in the Figures, which have been those used for the kinetic analysis, have been selected according to techniques recommended by Caballero et al. [타 ] so that the derivative of the points is calculated accurately and 
correctly, the points are equally spaced on a representation derivative of mass versus temperature and the fitting is simultaneous, with no variation of the kinetic constants, for at least three different heating rates.

With the objective of decreasing the great interrelation existing among the pre-exponential factor, the activation energy and the reaction order, the optimization was carried out using a "comparable kinetic constant" $\mathrm{k}_{\mathrm{i}}{ }^{*}$ instead of optimization of $\mathrm{k}_{\mathrm{i} 0}[\underline{16}]$.

$$
k_{i}^{i}=k_{i 0} \exp \left(\frac{-E_{i}}{R T_{\max }}\right)(0.64)^{n_{i}}
$$

In the previous equation, $T_{\max }$ is the temperature where maximum rate of decomposition is experimentally observed.

\section{Results and discussion}

\subsection{Fitting of thermogravimetric runs of materials studied (CWP, EWs and PCBs)}

Nine dynamic experiments were carried out by combining three different atmospheres $\left(\mathrm{N}_{2}, \mathrm{~N}_{2}: \mathrm{O}_{2}=9: 1\right.$ and $\left.\mathrm{N}_{2}: \mathrm{O}_{2}=4: 1\right)$ and three heating rates $\left(5,10\right.$ and $\left.20 \mathrm{~K} \mathrm{~min}^{-1}\right)$ from room temperature up to $1173 \mathrm{~K}$ for each material studied.

Figure 1 presents the decomposition of the materials in the absence of oxygen (pyrolysis). The decomposition of the EW, as mentioned, is given by the combination of two different materials: cover and insulation. In the Figure 1, also calculated curves are shown, that will be commented later. For all materials the mass loss curves move to the right (higher temperatures) when increasing heating rate, as expected. This behaviour has been observed by other authors that explained it using different arguments, but the only consideration of the kinetic law should be enough to explain this $[15,17]$. During the pyrolysis of the CWP sample, two different processes are clearly observed related to the decomposition of the hemicellulose and cellulose, and a long tail going until high temperatures related to the lignin devolatilization [18].

Figures 2 and 3 show the curves corresponding to the decomposition in the presence of $20 \%$ and $10 \%$ of oxygen, respectively, also with the corresponding calculated curves. The effect of the presence of oxygen is similar in all materials, accelerating the thermal decomposition (i.e. the curve is observed at lower temperatures compared to the pyrolysis) and producing the final reaction of the oxygen with the pyrolytic residue. The extent of this reaction is very much dependent on the material nature and the proportion of oxygen in the atmosphere.

In a previous work [7] it was shown that the decomposition of PCBs in the presence of oxygen presents an increase of the mass, due to the combination of the oxygen with the metal contained in the polymer. In the present situation, an increase in the mass is not observed because the PCB sample used was strictly non-metal contained.

To find a single set of kinetic parameters for pyrolytic and oxidant atmospheres for the different materials, the runs performed at different heating rates have been correlated simultaneously. The kinetic model proposed can be used for all these materials, but the decomposition of each material is formed parallel decompositions of different number of fractions (three in the case of CWP, three in oxidant atmospheres and two in pyrolysis for PCBs, two fractions for cover and only one fraction for insulation).

The kinetic parameters obtained are shown in Table 2. In order to bear in mind the presence of oxygen, values of the pre-exponential factors and activation energies have been allowed to vary respect to the pyrolytic conditions. In general, higher values of pre-exponential factors must be expected, in order to account for the acceleration of the process in the presence of oxygen, or lower values of activation energies. 
The calculated curves are shown in Figures 1 to 3, where the experimental curves are also presented. As can be seen in the Figures, the fitting obtained is very satisfactory to describe the behavior of the pyrolysis and combustion runs for each material.

[Table 2]

[Figures 1-3]

Note that for each material and atmosphere, the minimum number of fractions able to give a good fit was used. In this sense, only one fraction was necessary in the case of EW insulation material, two for the EW cover and PCBs pyrolysis. Furthermore, an optimization using first order reactions is done prior to let the order vary for all the materials studied, and it only was necessary an order different from unity for the synthetic materials and not for the biomass decomposition. As can be observed, the experimental and calculated curves are very similar indicating a good fit of the curves both in the absence and in the presence of oxygen.

The activation energies representing the pyrolytic decomposition of hemicellulose $\left(63 \mathrm{KJ} \cdot \mathrm{mol}^{-1}\right)$, cellulose $\left(137 \mathrm{KJ} \cdot \mathrm{mol}^{-}\right.$ $\left.{ }^{1}\right)$ and lignin $\left(49 \mathrm{KJ} \cdot \mathrm{mol}^{-1}\right)$ are in accordance with values found in literature [ㅁ] , but somewhat different bearing in mind the difference of the treatments given to the materials tested [13]. During thermal decomposition in the presence of oxygen, the activation energies (Table 2) increase for all three fractions, but a very high increase of the pre-exponential factors produces an acceleration of the process, as observed experimentally.

Respect to the kinetic constants obtained for the PCB decomposition, they are similar to those previously calculated for non-metallic PCBs [7], drawing attention the high reaction orders needed to fit the pyrolytic decomposition. Activation energies in the presence of oxygen are in general much lower than those in pyrolytic conditions, indicating the formation of species which promote the decomposition of the sample. The decomposition of the cover of EWs is explained using two different fractions, whereas the insulation plastic decomposition is described by only a fraction.

\subsection{Runs performed mixing CWP and EWs or PCBs}

In order to evaluate the possible synergistic effect combining biomass feedstocks and waste materials, different runs were performed by mixing CWP with halogen-free electrical wires (EWs) and printed circuit boards (PCBs). Specifically, runs at different heating rates with four different mixtures were performed: 20 and $50 \%$ of EW in CWP and also 20 and $50 \%$ of PCBs in CWP. The decomposition of these mixtures was studied in the absence and in the presence of oxygen at two different proportions $\left(\mathrm{N}_{2}: \mathrm{O}_{2}=9: 1\right.$ and $\left.\mathrm{N}_{2}: \mathrm{O}_{2}=4: 1\right)$.

Figures 4 to 6 show the main results. Only the curves performed at $10 \mathrm{~K} / \mathrm{min}$ are shown for the sake of simplicity, although 5 and $20 \mathrm{~K} / \mathrm{min}$ were also run (details can be found in Figures S1 to S3 of the supplementary material). Figure 4 presents the experimental decomposition curve in inert atmosphere of all four mixtures prepared, together with the calculated curves. The calculation was done by means of a weighted sum of the curves simulated using kinetics of the materials separately. Figures 5 and 6 show the corresponding curves for 10 and $20 \%$ oxygen in the atmosphere.

[Figures 4-6]

For all the mixtures prepared, the pyrolytic curves are very well explained by this simple sum of curves. This would mean that there is not interaction during the primary pyrolytic decomposition between the biomass and the waste materials. Contrarily, in oxidant atmospheres (Figures 5 and 6) the experimental curves are delayed respect to the calculated ones. This effect is more important as the heating rate is increased (See Figures in Supplementary Material) and also as the percentage of oxygen in the atmosphere is increased. 
This behaviour indicates that there exists an interaction between materials in the presence of oxygen, leading to decomposition that are slower than that observed with the materials separately. In the case mixture of PCBs the interaction is not as evident as in the case mixture of EWs. During the heating of the materials the primary decomposition products (those evolved from the solid material) may react between them (and oxygen if exists) forming species that are less volatile compared to the respective separate decomposition.

The behaviour observed would indicate that the decomposition products (including possible pollutants) is not affected by the presence of the other material in pyrolytic conditions, and probably this is also true for the combustion of PCBs. Nevertheless, a strong interaction is found in the mixtures between biomass and EWs, what produces important changes in the decomposition pattern, and hence in the decomposition products if were measured. At this moment we are working on our laboratory to prove this fact, comparing the emissions of the electrical wire determined previously [2] with those produced by the mixtures prepared. This is interesting to testing alternative fuels that would replace fossil fuels, for example in pellet stoves, in order to combine biomass and WEEE for the production of a high quality environmentally friendly Refuse Derived Fuel (RDF) at a reasonable price.

\section{Conclusions}

The thermal decomposition of different materials combining a biomass feedstock and WEEE has been studied. Kinetic models are proposed for the pyrolysis, gasification and combustion of CWP, halogen-free electrical wires (EWs) and printed circuit boards (PCBs) considering the effect of the heating rate in the decomposition at three different atmospheres.

Mixtures of these three materials have been prepared and tested for decomposition behavior, comparing the experimental curves and those obtained by a weighted sum of the curves simulated using kinetics of the materials separately. Differences are found specially in the case of EWs, indicating that there exists an interaction between materials in the presence of oxygen, leading to decomposition that are slower than that observed with the materials separately. In the case mixture of PCBs the interaction is not as evident as in the case mixture of EWs.

\section{Acknowledgements}

Support for this work was provided Spanish Ministry of Culture and Sport and by the CTQ2013-41006-R project from the Ministry of Economy and Competitiveness (Spain) and the PROMETEOII/2014/007 project from the Valencian Community Government (Spain).

\section{References}

1. Baldé CP, Wang, F., Kuehr, R., Huisman, J. The global e-waste monitor - 2014. United Nations University, IAS SCYCLE. 2015.

2. Conesa JA, Egea S, Moltó J, Ortuño N, Font R. Decomposition of two types of electric wires considering the effect of the metal in the production of pollutants. Chemosphere. 2013;91(2):118-23. doi:10.1016/j.chemosphere.2012.11.014. 3. Goosey M, Kellner R. Recycling technologies for the treatment of end of life printed circuit boards (PCBs). Circuit World. 2003;29(3):33-7. doi:10.1108/03056120310460801.

4. Kantarelis E, Donaj P, Yang W, Zabaniotou A. Sustainable valorization of plastic wastes for energy with environmental safety via High-Temperature Pyrolysis (HTP) and High-Temperature Steam Gasification (HTSG). J Hazard Mater. 2009;167(1-3):675-84.

5. Conesa JA, Moltó J, Font R, Egea S. Polyvinyl Chloride and Halogen-free Electric Wires Thermal Decomposition. Industrial \& Engineering Chemistry Research. 2010;49(22):11841-7. doi:10.1021/ie101265e.

6. Grønli MG, Várhegyi G, Di Blasi C. Thermogravimetric analysis and devolatilization kinetics of wood. Industrial and Engineering Chemistry Research. 2002;41(17):4201-8. 
7. Ortuño N, Moltó J, Egea S, Font R, Conesa JA. Thermogravimetric study of the decomposition of printed circuit boards from mobile phones. Journal of Analytical and Applied Pyrolysis. 2013.

8. Brebu M, Vasile C, Rovana Antonie S, Chiriac M, Precup M, Yang J et al. Study of the natural ageing of PVC insulation for electrical cables. Polym Degrad Stab. 2000;67(2):209-21.

9. Bene M, Plaek V, Matuschek G, Kettrup AA, Gyryov K, Emmerich WD et al. Lifetime simulation and thermal characterization of PVC cable insulation materials. J Therm Anal Calorim. 2005;82(3):761-8.

10. Dong C, Yang Y, Jin B, Horio M. The pyrolysis of sawdust and polyethylene in TG and U-shape tube reactor. Waste Management. 2007;27(11):1557-61. doi:10.1016/j.wasman.2006.10.021.

11. Kim MR, Buonomo EL, Bonelli PR, Cukierman AL. The thermochemical processing of municipal solid wastes: Thermal events and the kinetics of pyrolysis. Energy Sources, Part A: Recovery, Utilization and Environmental Effects. 2010;32(13):1207-14. doi:10.1080/15567030802665992.

12. Ortuño N, Moltó J, Conesa JA, Font R. Formation of brominated pollutants during the pyrolysis and combustion of tetrabromobisphenol A at different temperatures. Environ Pollut. 2014;191(0):31-7.

13. Várhegyi G, Grønli MG, Di Blasi C. Effects of Sample Origin, Extraction, and Hot-Water Washing on the Devolatilization Kinetics of Chestnut Wood. Industrial and Engineering Chemistry Research. 2004;43(10):2356-67.

14. Bach QV, Tran KQ, Skreiberg O, Khalil RA, Phan AN. Effects of wet torrefaction on reactivity and kinetics of wood under air combustion conditions. Fuel. 2014;137:375-83. doi:10.1016/j.fuel.2014.08.011.

15. Caballero JA, Conesa JA. Mathematical considerations for nonisothermal kinetics in thermal decomposition. Journal of Analytical and Applied Pyrolysis. 2005;73(1):85-100.

16. Martín-Gullón I, Gómez-Rico MF, Fullana A, Font R. Interrelation between the kinetic constant and the reaction order in pyrolysis. Journal of Analytical and Applied Pyrolysis. 2003;68-69:645-55. doi:10.1016/s0165-2370(03)000329.

17. Conesa JA, Rey L. Thermogravimetric and kinetic analysis of the decomposition of solid recovered fuel from municipal solid waste. J Therm Anal Calorim. 2015;120(2):1233. doi:10.1007/s10973-015-4396-4.

18. Conesa JA, Domene A. Biomasses pyrolysis and combustion kinetics through $\mathrm{n}$-th order parallel reactions. Thermochimica Acta. 2011;523(1-2):176-81. doi:10.1016/j.tca.2011.05.021.

\section{LIST OF TABLES AND FIGURES}

Table 1. Analysis of materials used.

Table 2. Kinetic parameter obtained for the pyrolysis and combustion models of materials studied.

Fig. 1. Evolution of the mass fraction of total sample for each material studied in inert atmosphere (pyrolysis).

Fig. 2. Evolution of the mass fraction of the total sample for each material studied in $20 \%$ oxygen $\left(\mathrm{N}_{2}: \mathrm{O}_{2}=4: 1\right)$.

Fig. 3. Evolution of the mass fraction of the total sample for each material studied in $10 \%$ oxygen $\left(\mathrm{N}_{2}: \mathrm{O}_{2}=9: 1\right)$

Fig. 4. Decomposition of the mixtures prepared at $10 \mathrm{~K} \mathrm{~min}^{-1}$ in pyrolytic atmosphere.

Fig. 5. Decomposition of the mixtures prepared at $10 \mathrm{~K} \mathrm{~min}^{-1}$ in the presence of $10 \%$ oxygen.

Fig. 6. Decomposition of the mixtures prepared at $10 \mathrm{~K} \mathrm{~min}^{-1}$ in the presence of $20 \%$ oxygen.

Figure S1 (Supplementary material). Decomposition of EWs/CWP mixtures in pyrolytic atmosphere at 5, 10 and $20 \mathrm{~K}$ $\min ^{-1}$.

Figure S2 (Supplementary material). Decomposition of EWs/CWP mixtures in nitrogen:oxygen=9:1 atmosphere at 5, 10 and $20 \mathrm{~K} \mathrm{~min}^{-1}$ 
Figure S3 (Supplementary material). Decomposition of EWs/CWP mixtures in nitrogen:oxygen=4:1 atmosphere at 5, 10 and $20 \mathrm{~K} \mathrm{~min}^{-1}$

Figure S4 (Supplementary material). Decomposition of PCBs/CWP mixtures in pyrolytic atmosphere at 5, 10 and $20 \mathrm{~K}$ $\min ^{-1}$

Figure S5 (Supplementary material). Decomposition of PCBs/CWP mixtures in nitrogen:oxygen=9:1 atmosphere at 5, 10 and $20 \mathrm{~K} \mathrm{~min}^{-1}$

Figure S6 (Supplementary material). Decomposition of PCBs/CWP mixtures in nitrogen:oxygen=4:1 atmosphere at 5, 10 and $20 \mathrm{~K} \mathrm{~min}^{-1}$ 
Elemental analysis *

\begin{tabular}{ccccc}
\hline mass \% & CWP & PCB & Cover & Insulation \\
\hline $\mathrm{C}$ & 51.56 & 25.61 & 29.81 & 15.29 \\
$\mathrm{H}$ & 6.53 & 2.22 & 7.13 & 3.74 \\
$\mathrm{~N}$ & n.d. & 0.91 & n.d. & n.d. \\
$\mathrm{S}$ & n.d. & n.d. & n.d. & n.d. \\
O and ash (by & 41.92 & 71.26 & & \\
difference) & & & 63.06 & 80.97 \\
\hline Ash ** & 1.13 & 56.67 & 44.07 & 78.48 \\
\hline $\mathrm{O}$ & Fluorescence analysis & & \\
$\mathrm{Ca}$ & 15.46 & 26.81 & 28.34 & 40.31 \\
$\mathrm{Si}$ & 14.76 & 11.76 & n.d. & 0.17 \\
$\mathrm{Al}$ & 3.53 & 15.83 & 2.86 & 33.68 \\
$\mathrm{~K}$ & 1.91 & 4 & 27.00 & 0.96 \\
$\mathrm{Fe}$ & 1.65 & 0.14 & n.d. & 0.62 \\
$\mathrm{P}$ & 1.44 & 0.15 & 0.09 & 0.15 \\
$\mathrm{Mg}$ & 1.29 & 0.04 & n.d. & n.d. \\
$\mathrm{Na}$ & 0.98 & 0.16 & 1.58 & 0.12 \\
$\mathrm{~S}$ & 0.46 & 0.05 & n.d. & n.d. \\
$\mathrm{Cl}$ & 0.28 & 0.02 & n.d. & 0.06 \\
$\mathrm{Sr}$ & 0.12 & 0.1 & n.d. & n.d. \\
$\mathrm{Cu}$ & 0.03 & 0.1 & n.d. & n.d. \\
$\mathrm{Zn}$ & n.d. & 0.01 & n.d. & 0.06 \\
$\mathrm{Br}$ & n.d. & nd & 2.54 & 3.39 \\
$\mathrm{Ti}$ & n.d. & 11.8 & n.d. & n.d. \\
$\mathrm{Ce}$ & n.d. & 0.28 & n.d. & 1.06 \\
$\mathrm{Cr}$ & n.d. & n.d. & n.d. & 0.40 \\
& n.d. & n.d. & n.d. & n.d. \\
\hline & & & &
\end{tabular}

nd $=$ not detected

*By combustion in pure oxygen at $1000^{\circ} \mathrm{C}$

**Determined by norm UNE-EN-14775:2009 at 550 ${ }^{\circ} \mathrm{C}$ 
Table 2. Kinetic parameters obtained for the pyrolysis and combustion models of materials studied.

\begin{tabular}{|c|c|c|c|c|}
\hline \multicolumn{5}{|c|}{ Crushed wood pellets, CWP } \\
\hline Reaction & $k_{\mathrm{i} 0} / \mathrm{s}^{-1}$ & $E_{\mathrm{i}} / \mathrm{KJ} \mathrm{mol}^{-1}$ & $n_{\mathrm{i}}$ & $c_{\mathrm{Si} 0}$ \\
\hline \multicolumn{5}{|l|}{ Pyrolysis } \\
\hline 1 & $1.07 \cdot 10^{3}$ & 63.8 & 1.00 & 0.394 \\
\hline 2 & $1.31 \cdot 10^{9}$ & 137.0 & 1.00 & 0.325 \\
\hline 3 & $7.79 \cdot 10^{-1}$ & 49.0 & 1.00 & 0.124 \\
\hline \multicolumn{5}{|c|}{$\mathrm{N}_{2}: \mathrm{O}_{2}=4: 1$} \\
\hline 1 & $1.89 \cdot 10^{6}$ & 117.4 & 1.00 & 0.362 \\
\hline 2 & $1.95 \cdot 10^{10}$ & 144.3 & 1.00 & 0.170 \\
\hline 3 & $8.78 \cdot 10^{3}$ & 73.2 & 1.00 & 0.443 \\
\hline \multicolumn{5}{|c|}{$\mathrm{N}_{2}: \mathrm{O}_{2}=9: 1$} \\
\hline 1 & $1.35 \cdot 10^{6}$ & 117.4 & 1.00 & 0.359 \\
\hline 2 & $2.01 \cdot 10^{10}$ & 144.3 & 1.00 & 0.451 \\
\hline 3 & $3.17 \cdot 10^{4}$ & 73.2 & 1.00 & 0.165 \\
\hline \multicolumn{5}{|c|}{ PCBs } \\
\hline Reaction & $k_{\mathrm{i} 0} / \mathrm{s}^{-1}$ & $E_{\mathrm{i}} / \mathrm{KJ} \mathrm{mol}^{-1}$ & $n_{\mathrm{i}}$ & $c_{\mathrm{Si} 0}$ \\
\hline \multicolumn{5}{|l|}{ Pyrolysis } \\
\hline 1 & $8.71 \cdot 10^{18}$ & 236.2 & 4.75 & 0.110 \\
\hline 2 & $8.81 \cdot 10^{24}$ & 302.1 & 2.04 & 0.239 \\
\hline \multicolumn{5}{|c|}{$\mathrm{N}_{2}: \mathrm{O}_{2}=4: 1$} \\
\hline 1 & $6.50 \cdot 10^{12}$ & 168.4 & 1.00 & 0.134 \\
\hline 2 & $2.84 \cdot 10^{20}$ & 258.8 & 2.80 & 0.100 \\
\hline 3 & $1.60 \cdot 10^{6}$ & 128.2 & 1.00 & 0.201 \\
\hline \multicolumn{5}{|c|}{$\mathrm{N}_{2}: \mathrm{O}_{2}=9: 1$} \\
\hline 1 & $5.57 \cdot 10^{12}$ & 168.4 & 1.00 & 0.123 \\
\hline 2 & $2.18 \cdot 10^{20}$ & 258.8 & 2.80 & 0.120 \\
\hline 3 & $7.67 \cdot 10^{5}$ & 128.2 & 1.06 & 0.173 \\
\hline \multicolumn{5}{|c|}{ Cover } \\
\hline Reaction & $k_{\mathrm{i} 0} / \mathrm{s}^{-1}$ & $E_{\mathrm{i}} / \mathrm{KJ} \mathrm{mol}^{-1}$ & $n_{\mathrm{i}}$ & $c_{\mathrm{Si} 0}$ \\
\hline \multicolumn{5}{|l|}{ Pyrolysis } \\
\hline 1 & $1.29 \cdot 10^{22}$ & 339.8 & 1.44 & 0.301 \\
\hline 2 & $2.52 \cdot 10^{17}$ & 210.9 & 6.63 & 0.283 \\
\hline \multicolumn{5}{|c|}{$\mathrm{N}_{2}: \mathrm{O}_{2}=4: 1$} \\
\hline 1 & $7.94 \cdot 10^{17}$ & 217.2 & 7.64 & 0.428 \\
\hline 2 & $8.66 \cdot 10^{23}$ & 350.5 & 3.19 & 0.157 \\
\hline \multicolumn{5}{|c|}{$\mathrm{N}_{2}: \mathrm{O}_{2}=9: 1$} \\
\hline 1 & $3.87 \cdot 10^{13}$ & 210.3 & 2.58 & 0.323 \\
\hline 2 & $1.48 \cdot 10^{15}$ & 185.2 & 3.91 & 0.258 \\
\hline
\end{tabular}




\begin{tabular}{rllll}
\hline Reaction & $k_{\mathrm{i} 0} / \mathrm{s}^{-1}$ & $E_{\mathrm{i}} / \mathrm{KJ} \mathrm{mol}^{-1}$ & $n_{\mathrm{i}}$ & $c_{\mathrm{Si} 0}$ \\
\hline Pyrolysis & & & 1.90 & $0.418^{*}$ \\
1 & $3.35 \cdot 10^{6}$ & 138.2 & & \\
$\mathrm{~N}_{2}: \mathrm{O}_{2}=4: 1$ & & & & \\
1 & $3.19 \cdot 10^{6}$ & 124.5 & 1.74 & $0.377^{*}$ \\
$\mathrm{~N}_{2}: \mathrm{O}_{2}=9: 1$ & & & & \\
1 & $6.77 \cdot 10^{5}$ & 116.1 & 1.99 & $0.350 *$ \\
\hline
\end{tabular}

* fixed value (not considered as a parameter to be fitted) 


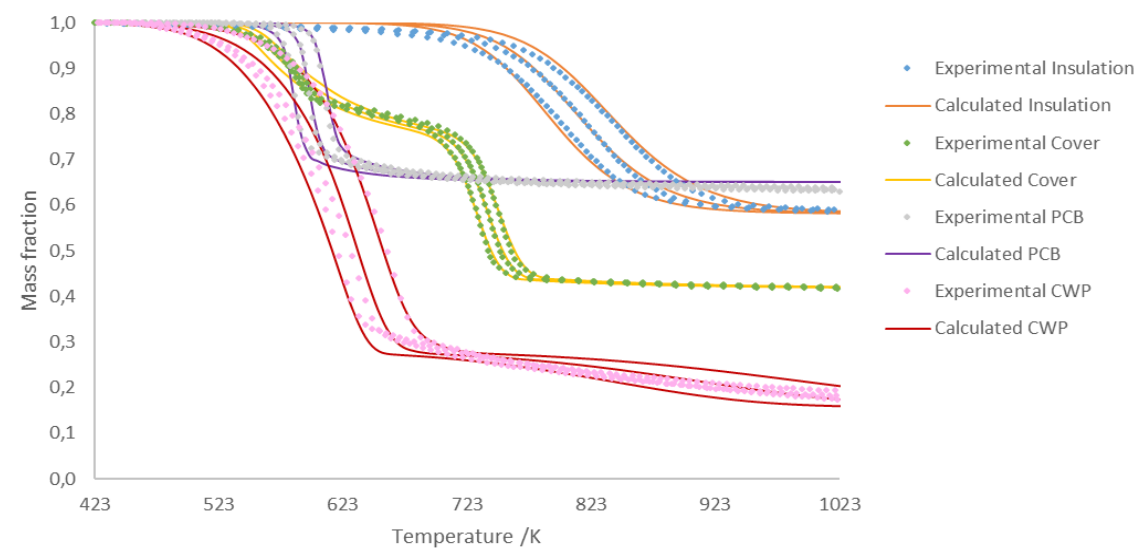

Fig. . Evolution of the mass fraction of total sample for each material studied in inert atmosphere (pyrolysis).

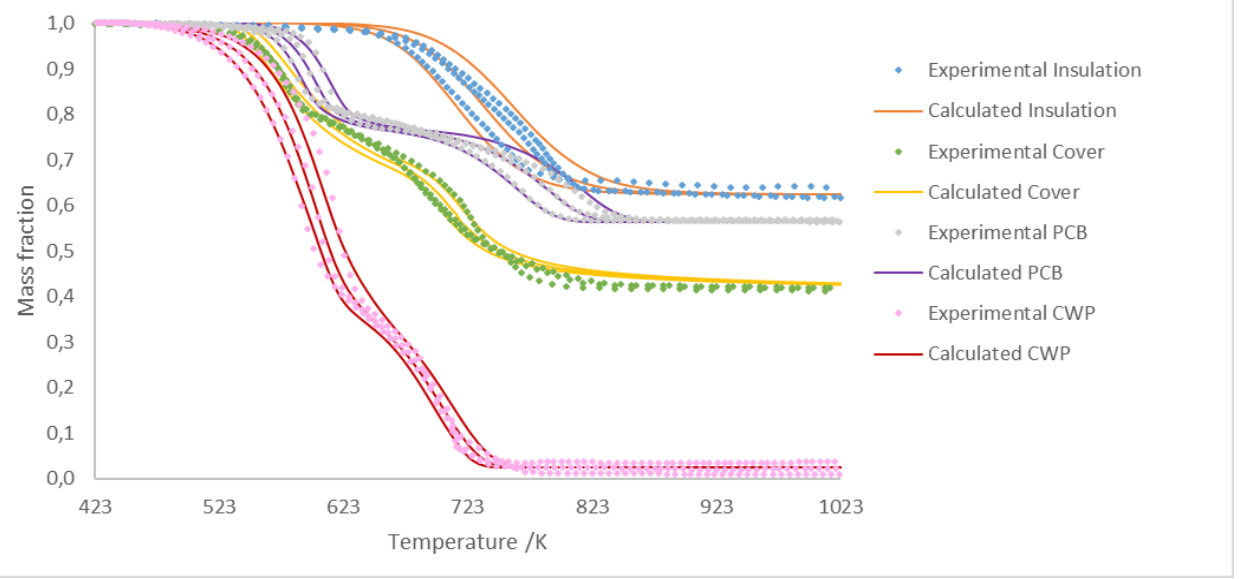

Fig. . Evolution of the mass fraction of the total sample for each material studied in $20 \%$ oxygen $\left(\mathrm{N}_{2}: \mathrm{O}_{2}=4: 1\right)$.

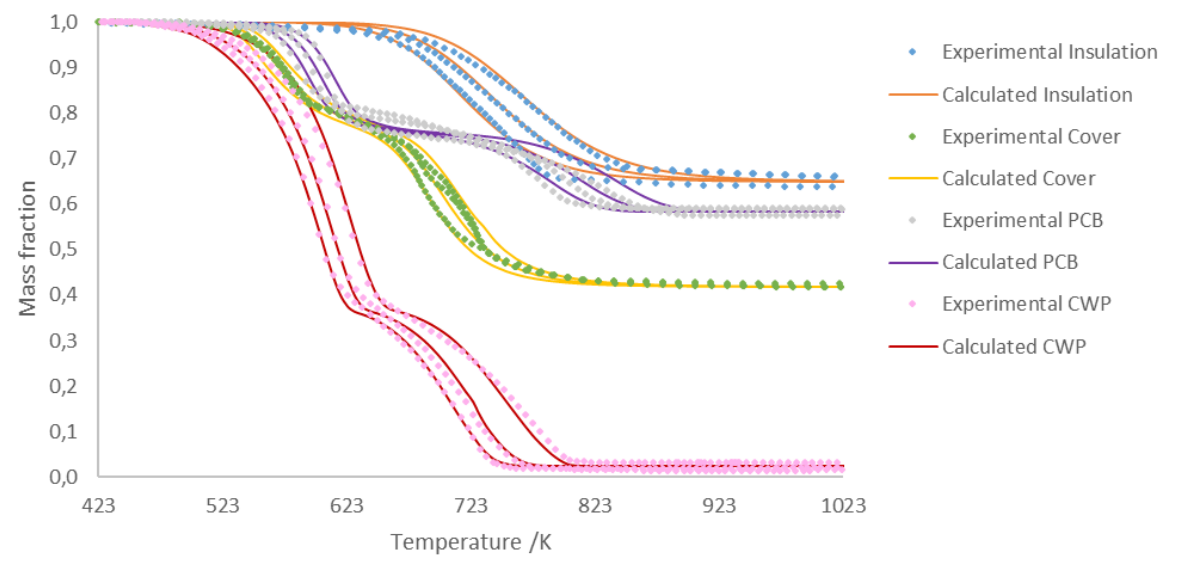

Fig. . Evolution of the mass fraction of the total sample for each material studied in $10 \%$ oxygen $\left(\mathrm{N}_{2}: \mathrm{O}_{2}=9: 1\right)$. 


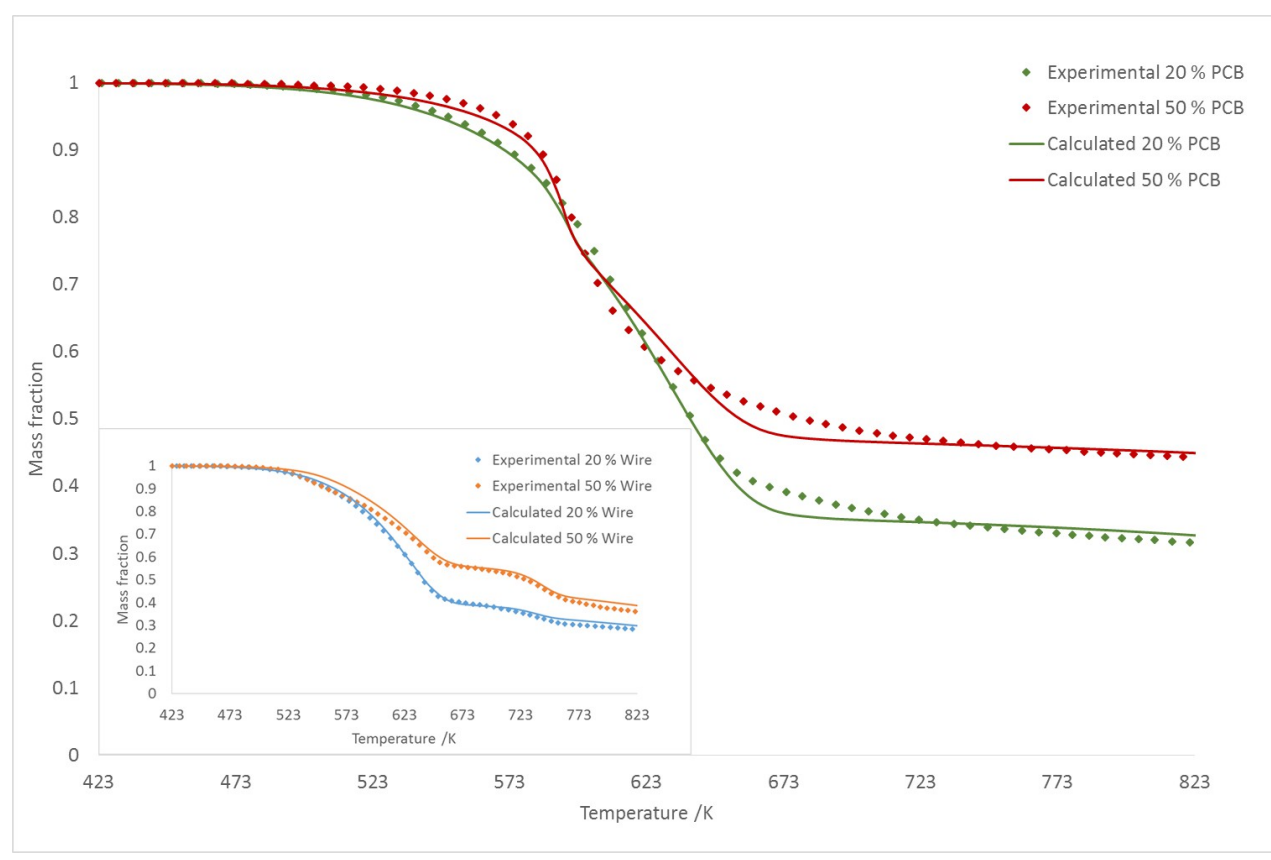

Fig. . Decomposition of the mixtures prepared at $10 \mathrm{~K} \mathrm{~min}^{-1}$ in pyrolytic atmosphere.

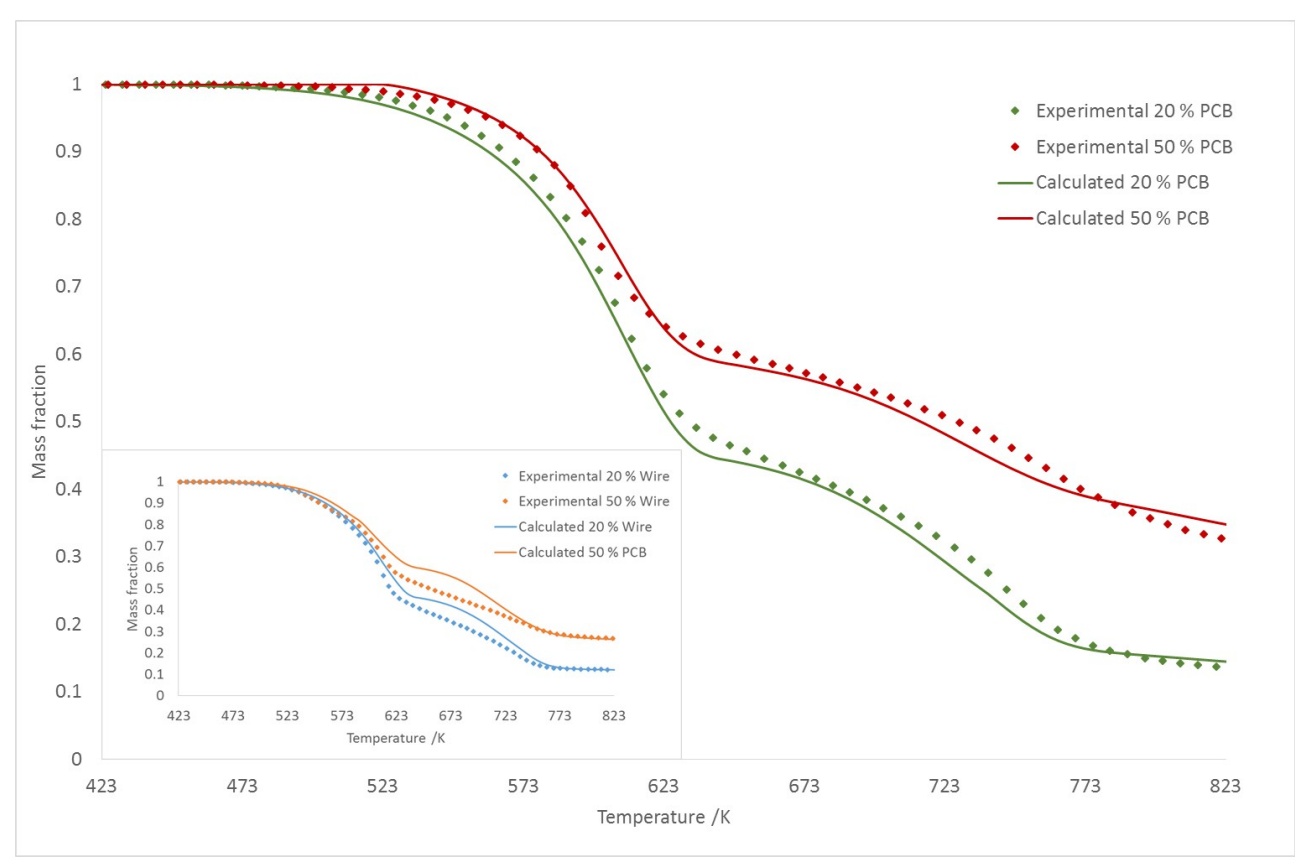

Fig. . Decomposition of the mixtures prepared at $10 \mathrm{~K} \mathrm{~min}^{-1}$ in the presence of $10 \%$ oxygen. 


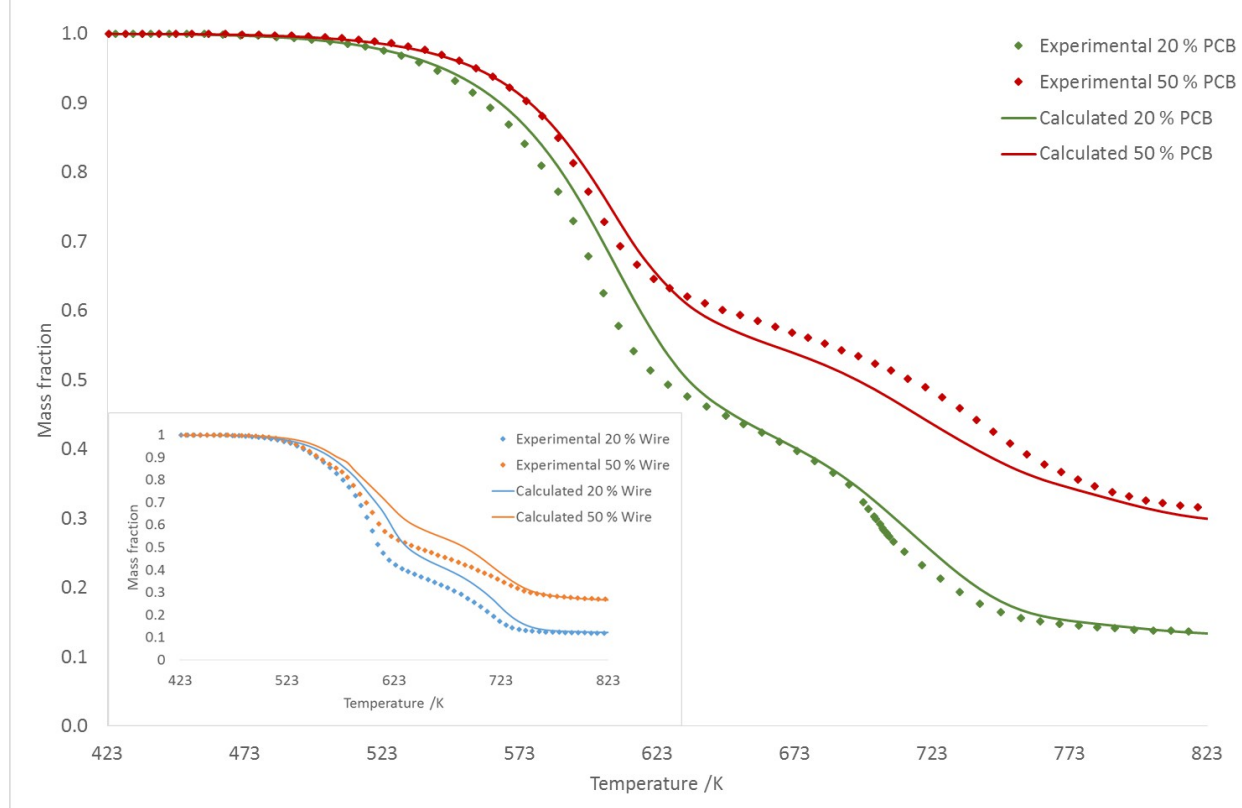

Fig. . Decomposition of the mixtures prepared at $10 \mathrm{~K} \mathrm{~min}^{-1}$ in the presence of $20 \%$ oxygen. 

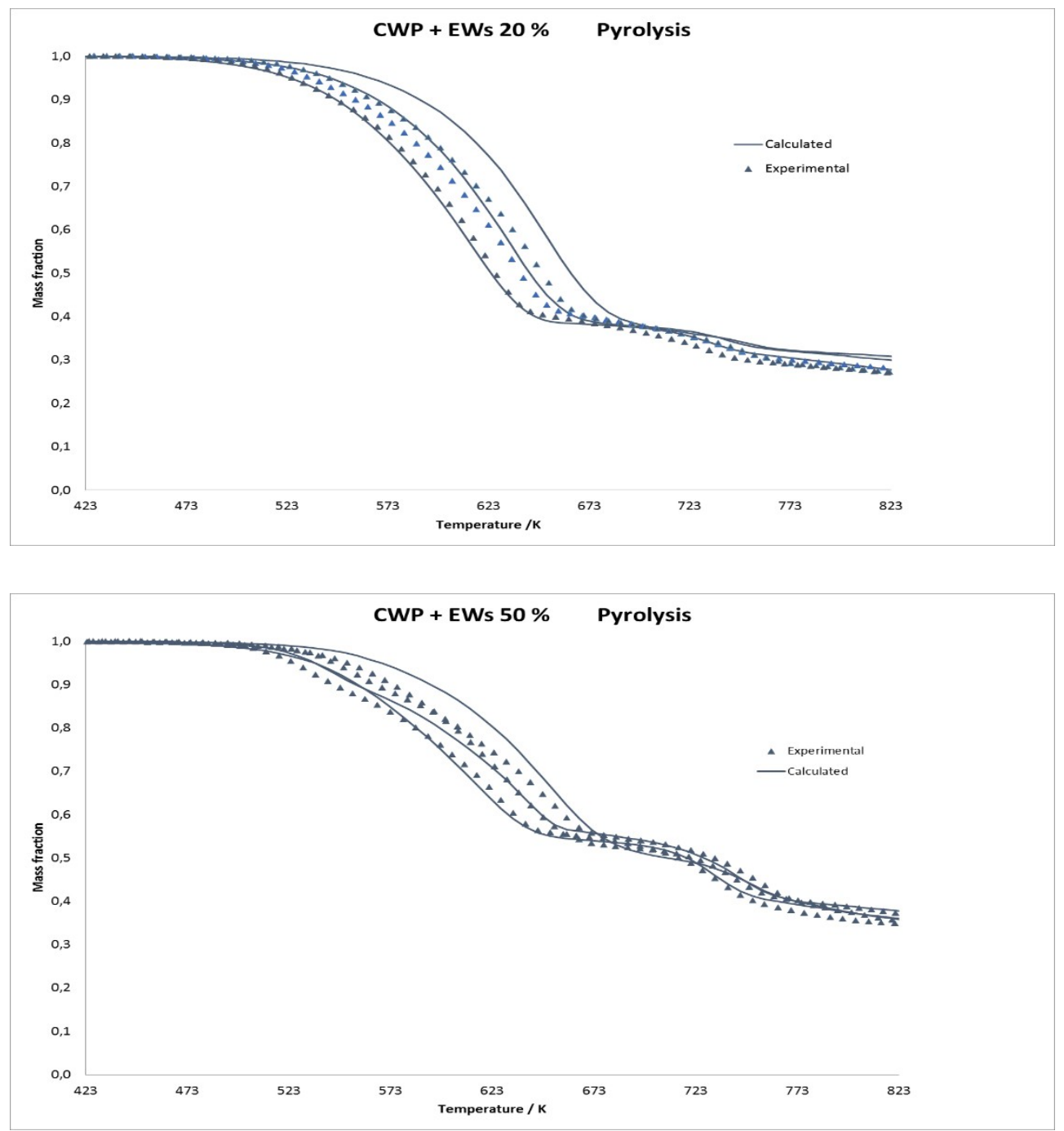

Figure S1 (Supplementary material). Decomposition of EWs/CWP mixtures in pyrolytic atmosphere at 5, 10 and $20 \mathrm{~K}$ $\min ^{-1}$. 

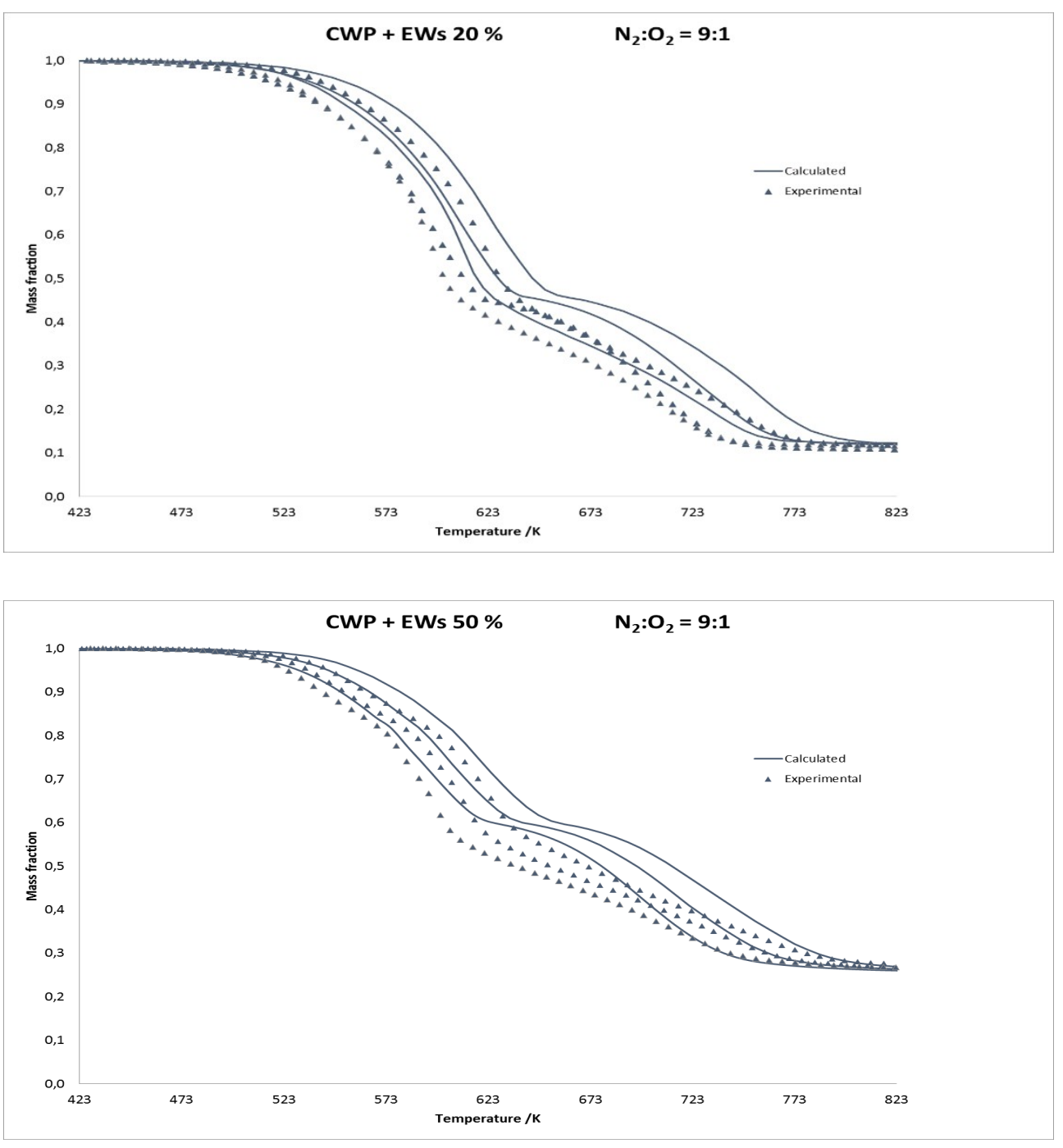

Figure S2 (Supplementary material). Decomposition of EWs/CWP mixtures in nitrogen:oxygen=9:1 atmosphere at 5, 10 and $20 \mathrm{~K} / \mathrm{min}$ 

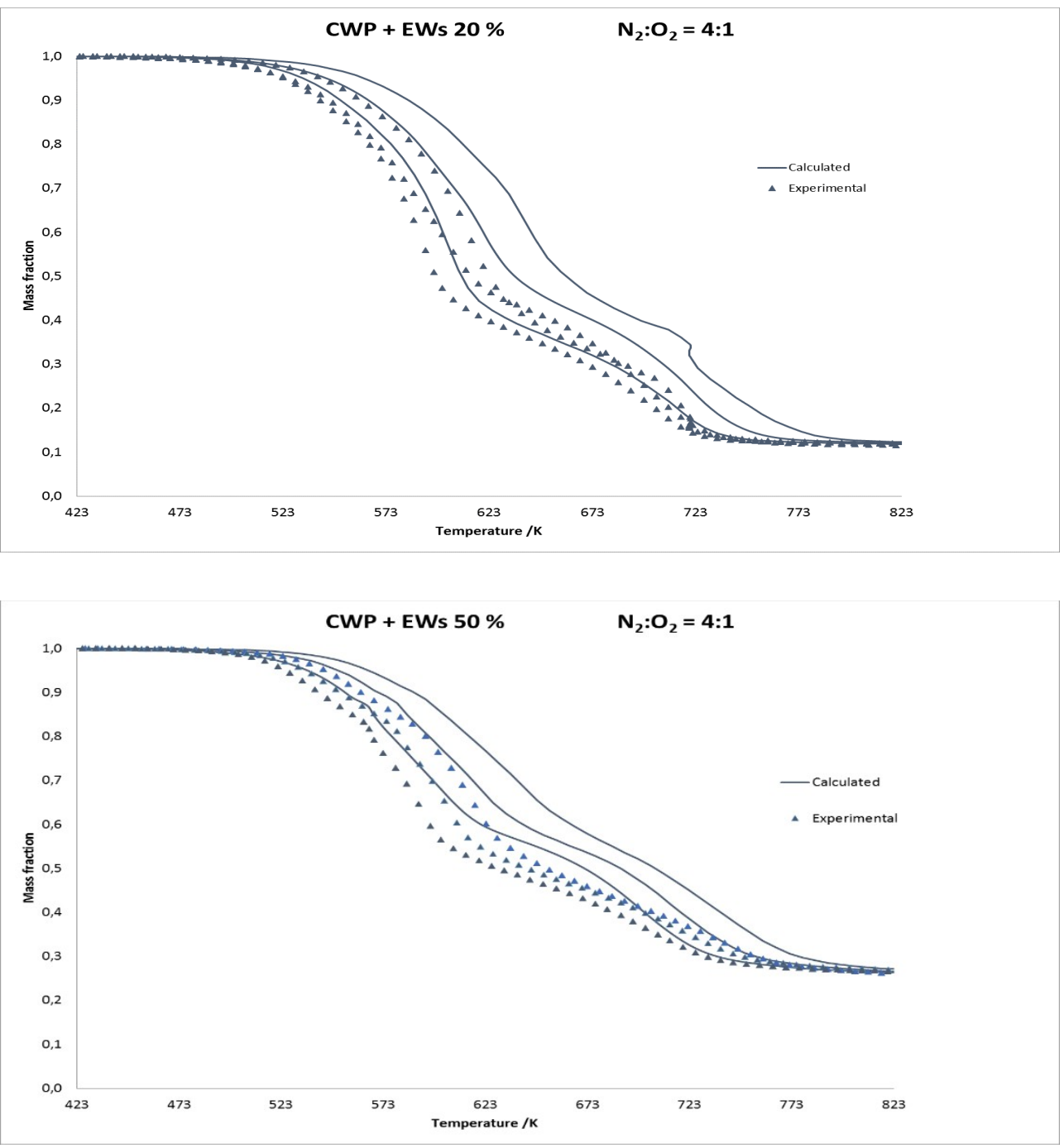

Figure S3 (Supplementary material). Decomposition of EWs/CWP mixtures in nitrogen:oxygen=4:1 atmosphere at 5, 10 and $20 \mathrm{~K} \mathrm{~min}^{-1}$ 

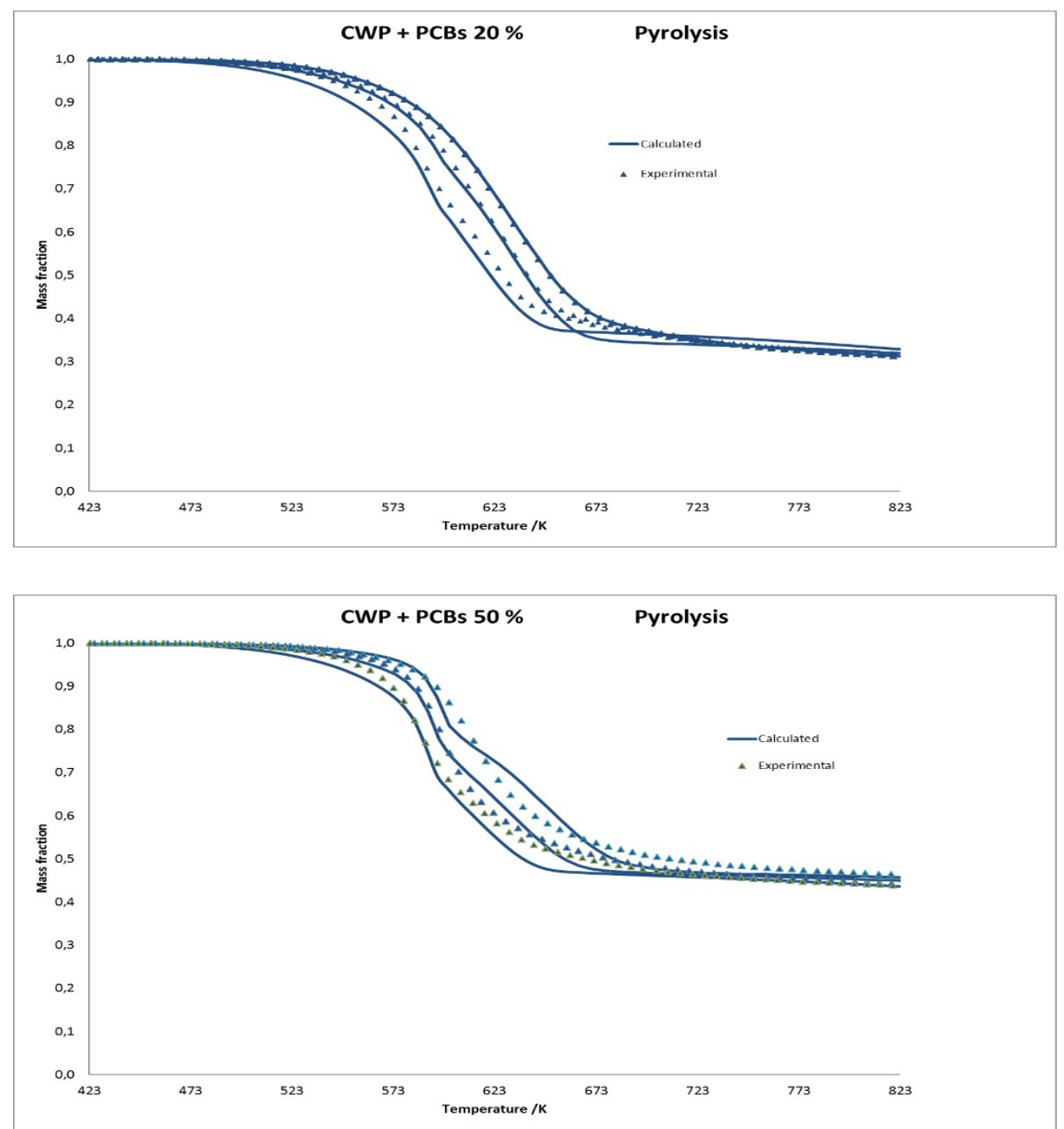

Figure S4 (Supplementary material). Decomposition of PCBs/CWP mixtures in pyrolytic atmosphere at 5, 10 and $20 \mathrm{~K}$ $\min ^{-1}$ 

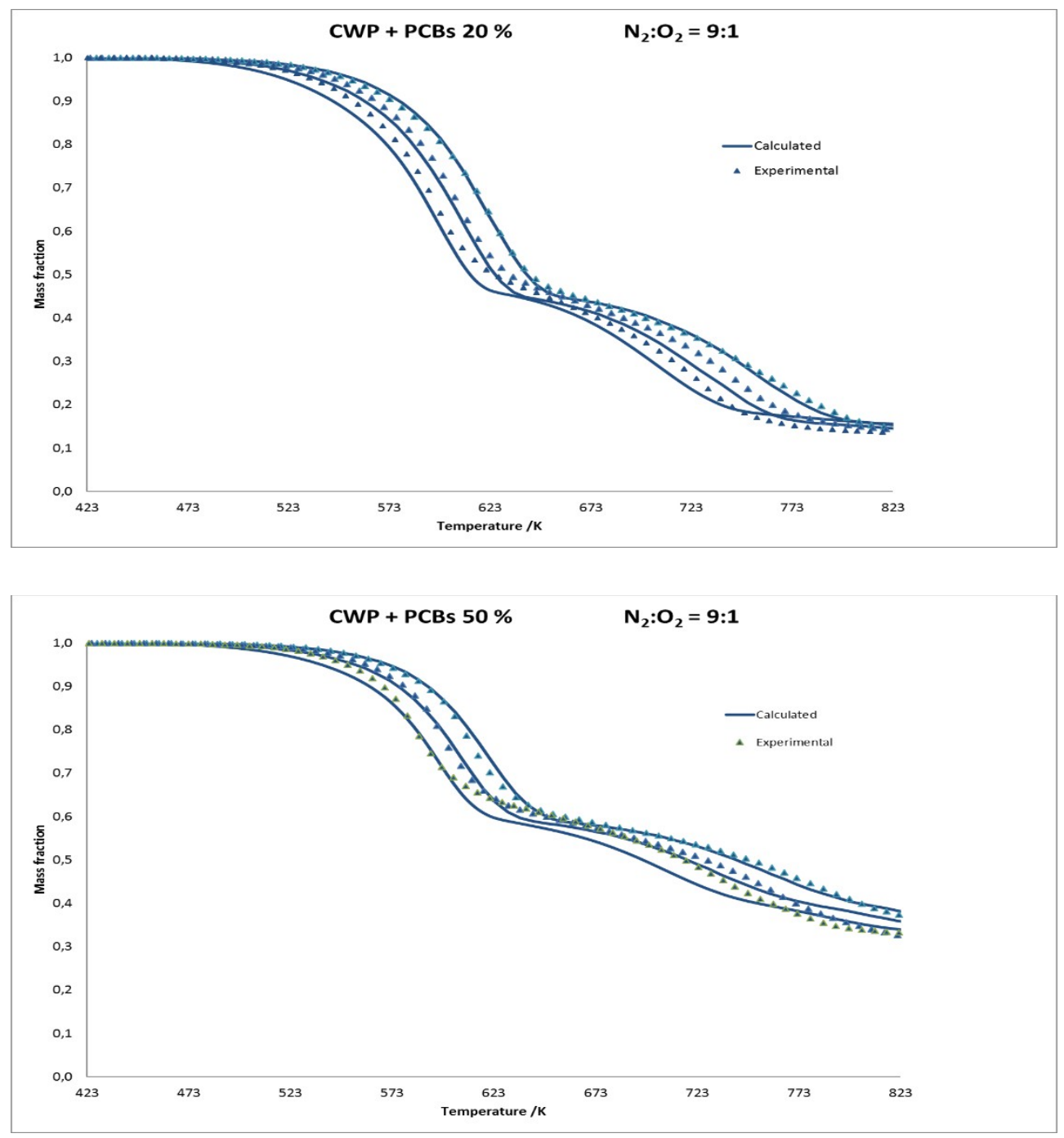

Figure S5 (Supplementary material). Decomposition of PCBs/CWP mixtures in nitrogen:oxygen=9:1 atmosphere at 5, 10 and $20 \mathrm{~K} \mathrm{~min}^{-1}$ 

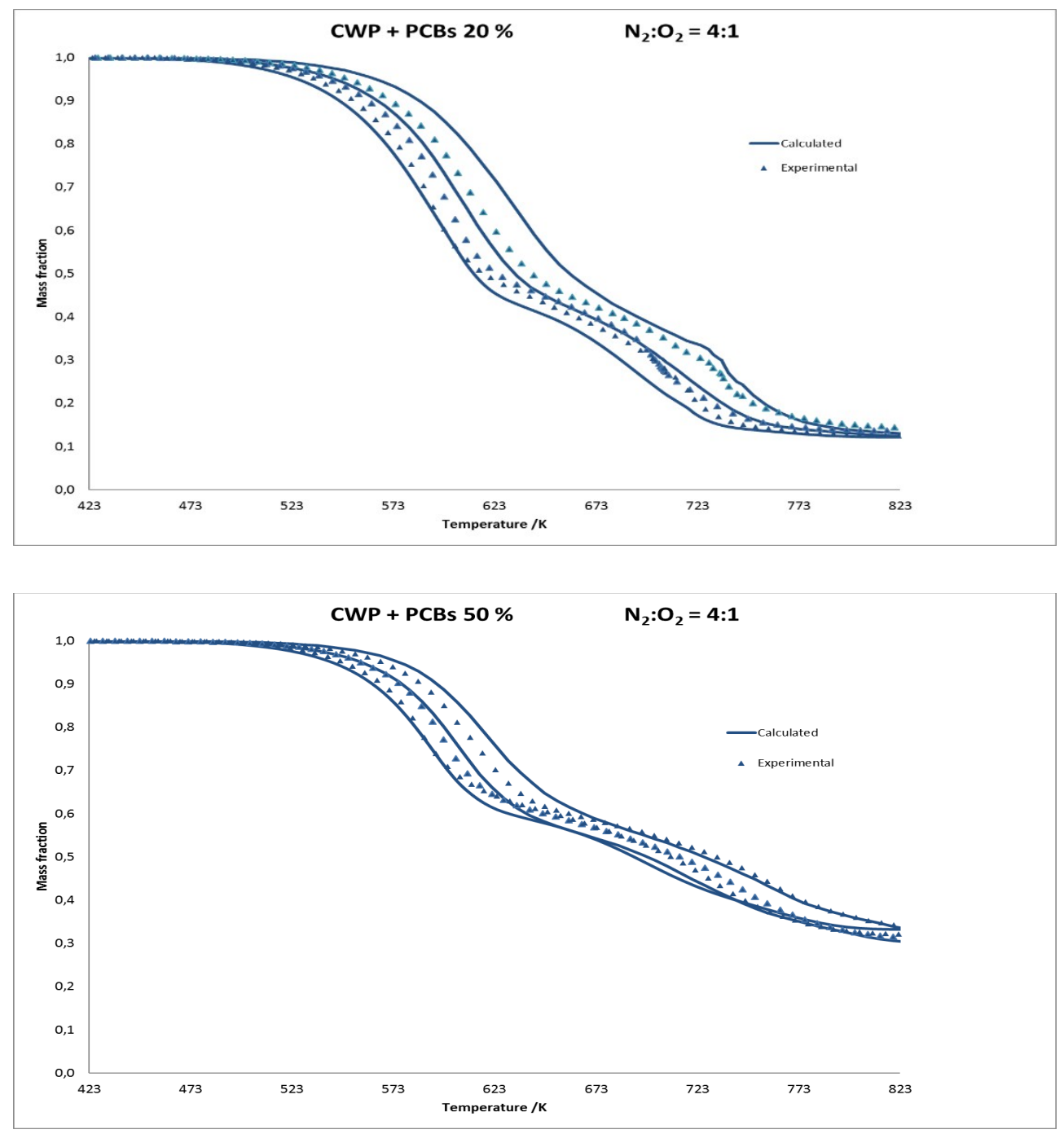

Figure S6 (Supplementary material). Decomposition of PCBs/CWP mixtures in nitrogen:oxygen=4:1 atmosphere at 5, 10 and $20 \mathrm{~K} \mathrm{~min}^{-1}$ 